Article

\title{
Data Transmission Direction Based Routing Algorithm for Improving Network Performance of IoT Systems
}

\author{
Kyeong Mi Noh ${ }^{1}\left(\right.$, Jong Hyuk Park ${ }^{2}\left(\mathbb{D}\right.$ and Ji Su Park ${ }^{3, *}$ \\ 1 Department of Information Communication Media Engineering, Graduate School of Nano It Design Fusion, \\ Seoul National University of Science \& Technology (SeoulTech), Seoul 01811, Korea; nkkaeng@knou.ac.kr \\ 2 Department of Computer Science and Engineering, Seoul National University of Science \& Technology \\ (SeoulTech), Seoul 01811, Korea; jhpark1@seoultech.ac.kr \\ 3 Department of Computer Science and Engineering, Jeonju University, Jeonju 55069, Korea \\ * Correspondence: jisupark@jj.ac.kr
}

Received: 16 April 2020; Accepted: 25 May 2020; Published: 29 May 2020

\begin{abstract}
With the continuous development of wireless communication technology, the Internet of Things (IoT) is being used in a wide range of fields. The IoT collects and exchanges large amounts of data with objects, either tangible or intangible, such as sensors or physical devices, connected to the Internet. Wireless sensor networks (WSNs) are components of IoT systems. WSNs are used in various IoT systems, such as monitoring, tracking, and detection systems, to extract relevant information and deliver it to users. WSNs consist of sensor nodes with low power, low cost, and multiple functions. Because sensor nodes have limited resources, such as power and memory, a reduction in the energy efficiency of the sensor nodes in WSNs will lead to a decrease in wireless network performance and an increase in packet loss, which affects IoT system performance. Therefore, this study aimed to find an energy-efficient routing method that extends the lifetime of WSNs by minimizing the battery use of sensor nodes to improve the network performance of IoT systems. Conserving energy from sensor nodes and increasing network throughput in WSNs involves having protocols. The low-energy adaptive clustering hierarchy (LEACH ) protocol is a well-known hierarchical routing protocol in WSNs that constructs clusters and transmits data. LEACH increases energy efficiency by transmitting data from sensor nodes to the base station (BS) through the cluster head. It is widely adopted in the WSN network field, and many protocols are being studied to improve cluster header selection and data transmission to increase the energy efficiency of sensor nodes. In this study, we attempted to improve energy efficiency by removing unnecessary energy from LEACH. In LEACH, when the sensor node is located between the BS and the cluster head, the sensor node transmits data to the cluster head in the opposite direction of the BS. The data sent to the cluster head are transmitted in the direction of the BS. Thus, transmission in the opposite direction consumes unnecessary energy and affects the WSN performance of IoT systems. In this study, we propose a D-LEACH (direction-based LEACH) protocol based on the received signal strength indicator (RSSI) that improves the efficiency of transmission energy considering the data transmission direction of sensor nodes. D-LEACH aims to balance the energy of the sensor nodes and improve the performance of WSNs in the IoT system by reducing unnecessary energy consumption caused by reverse transmission considering the data transmission direction of the sensor nodes. In the course of the paper, we refer to the routing protocol of WSNs to improve network performance and describe LEACH. We also explain the D-LEACH protocol proposed in this paper and confirm the performance improvement of WSNs in an IoT system through simulation.
\end{abstract}

Keywords: IoT; wireless sensor networks; D-LEACH; routing protocol; network performance 


\section{Introduction}

The Internet of Things (IoT) connects objects, people, various systems, and information resources to manage information from the real and virtual world and provide intelligent services. IoT features objects that recognize a situation, and many IoT systems are required to provide services related to objects that know their surroundings and are associated with them.

With the development of wireless communication technology and micro-electronic mechanical systems (MEMS) technology, the IoT can be used in various applications that require wireless communication technologies, such as transportation devices, healthcare, military, and smart homes [1-4]. Wireless sensor networks (WSNs) can be considered an Internet extension of the physical environment in IoT and are a component of the IoT system and IoT community [5]. WSNs consist of sensor nodes that have limitations regarding battery lifetime. Sensor nodes can detect the surrounding environment, configure the network, and transmit data, and data from sensor nodes can be sent to the user via the base station (BS) [6-9]. The battery lifetime of the sensor node is the most important factor to consider when designing WSNs. Therefore, improving the network performance of IoT systems must be the focus, by designing energy-efficient WSN protocols.

A sensor node is a hardware device that senses the environment, processes data, and transmits data. The sensor node consists of a sensing unit, processing unit, communication unit, and power unit, as shown in Figure 1. Depending on the application, it may also have a location finding system, mobilizer, and power generator.

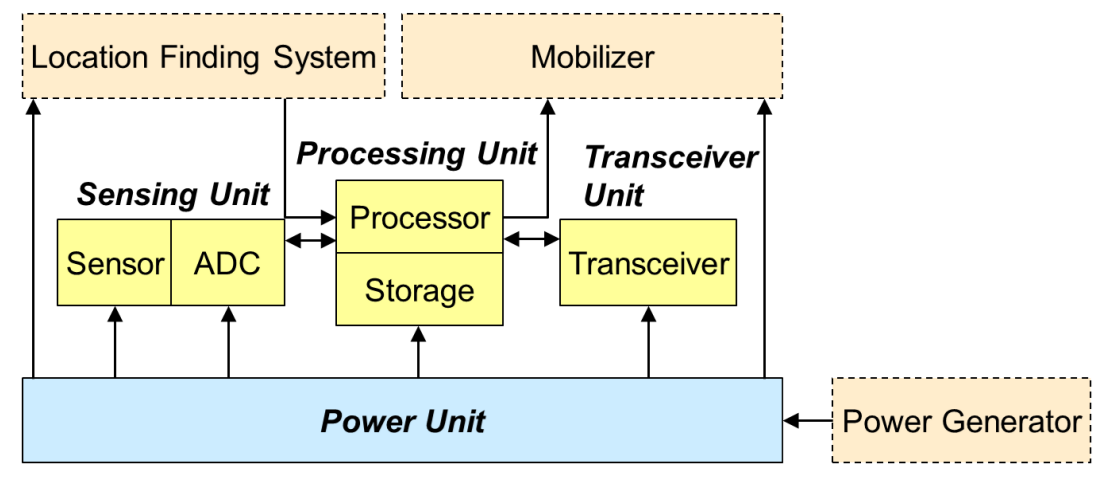

Figure 1. The components of a sensor node.

The sensor nodes collect the sensed data and transmit them to the BS located between the sensor nodes. Sensor nodes are small, are lightweight, and have low production costs, they also have many limitations such as energy, memory capacity, and processing speed [10-12]. These constraints affect the communication distance and data processing speed between sensor nodes. WSNs are used in various fields, such as terrain, climate, military, and ecological monitoring. The sensor node is randomly arranged according to the environment and purpose or is placed at a designated location. The deployed sensor node constructs the network and transmits the collected information to the BS. During this operation, data communication should be performed in an energy-efficient manner. The routing protocol of WSNs can extend the lifetime of WSNs by designing and implementing the efficient use of the energy of the sensor node [13].

The routing protocol of WSNs can be divided into flat network routing, hierarchical routing, and location-based routing depending on the network structure. The hierarchical routing protocol divides sensor nodes into clusters and has a hierarchy of cluster headers $(\mathrm{CHs})$ and member nodes in the cluster. Clustering in WSNs is a protocol for reducing the energy usage of sensor nodes. Cluster-based routing increases energy efficiency, reduces transmission latency, and improves network scalability [14]. Clustering selects a representative node called a cluster header among the sensor nodes scattered in the field, and the $\mathrm{CH}$ collects data from peripheral nodes and transmits them to the BS. Sensor nodes can conserve energy by sending data to the BS via the $\mathrm{CH}$ [15]. Therefore, by selecting 
the $\mathrm{CH}$ among the sensor nodes, energy usage can be reduced and the life of WSNs can be extended. The low-energy adaptive clustering hierarchy (LEACH) is a cluster-based hierarchical routing protocol for cluster configuration and data transmission. LEACH saves the transmission energy of the sensor node by transmitting data after configuring the cluster [16-20]. Many studies have been conducted to improve $\mathrm{CH}$ elective and data transmission methods in LEACH. Meanwhile, in this study, we focused on reducing energy loss caused by reverse transmission in LEACH.

In $\mathrm{LEACH}$, when the sensor node is between the BS and the $\mathrm{CH}$, the sensor node transmits data to the $\mathrm{CH}$. At this time, data transmission occurs in the opposite direction of the BS. Energy dissipation associated with data transmission from the sensor node to the $\mathrm{CH}$ affects network performance. Figure 2 shows the reverse transmission.

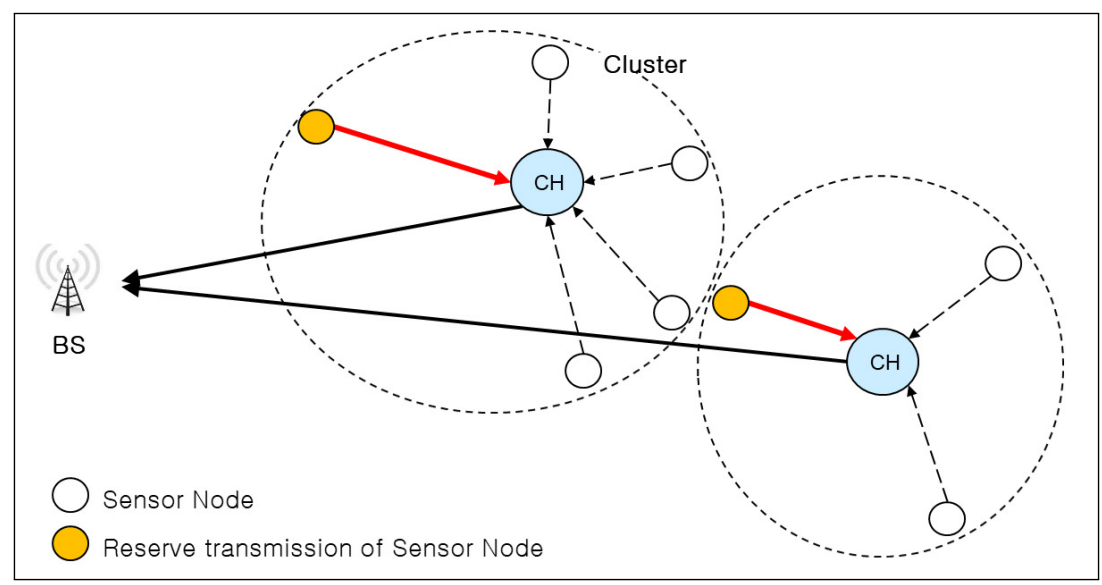

Figure 2. Reverse transmission of sensor nodes.

In this study, to improve the network performance of IoT systems, we attempted to increase the energy efficiency by eliminating the reverse transmission of sensor nodes. We propose a direction-based LEACH (D-LEACH) based on the transmission direction of the sensor node. D-LEACH reduces the unnecessary energy consumption of LEACH by selecting the direction of data transmission.

D-LEACH consists of four phases: layer configuration phase, probability calculation phase for $\mathrm{CH}$ selection, setup phase, and steady-state phase. D-LEACH divides the WSNs into several layers using the relative distance between the BS and sensor nodes. The number of sensor nodes in each layer is different for each layer. The top layer has the largest number of sensor nodes and the lowest layer has the smallest number of sensor nodes. Each layer calculates the $\mathrm{CH}$ selection probability. The layer with many sensor nodes has a high probability of $\mathrm{CH}$ selection. The sensor nodes of each layer select the $\mathrm{CH}$ using the selection probability. The sensor node becomes a member node of the $\mathrm{CH}$ having the strongest signal intensity among the $\mathrm{CHs}$ located closer to the BS than itself. The sensor node uses the relative distance to determine the direction of the data transmission. It transmits the data to the location of the BS using direct transmission, single-hop transmission or multi-hop transmission.

The remainder of this paper is organized as follows. Section 2 discusses the cluster-based routing protocol, and Section 3 discusses LEACH. Section 4 introduces the proposed energy-efficient routing protocol, and Section 5 analyzes and compares the performance of the proposed routing protocol. Finally, Section 6 concludes the paper.

\section{Related Work}

\subsection{Cluster-Based Routing Protocol}

The energy-efficient design of WSNs in IoT systems is complicated owing to the energy constraints of sensor nodes. The energy efficiency of WSNs is an important issue in the evaluation of IoT performance. To achieve energy efficiency for sensor nodes, many routing algorithms based on clustering have been proposed [21,22]. 
LEACH is a well-known cluster-based routing protocol for WSNs [23]. WSNs select CHs at regular intervals, and each sensor node has the same probability of becoming a $\mathrm{CH}$. The sensor node transmits data to the $\mathrm{BS}$ through the $\mathrm{CH}$, thereby increasing the energy efficiency of the sensor node to extend the lifetime of the network. Many protocols have been proposed that improve performance based on LEACH.

A clustering-based routing algorithm in IoT-aware wireless mesh networks configures clustering by selecting adjacent clusters to avoid flooding the network [24]. It also increases network throughput by analyzing undistributed routes.

The k-means clustering algorithm proposes an optimized cluster structure using the weighted function of the k-means clustering algorithm [25]. The weighted function evaluation determines whether to split or merge the cluster structure. The $\mathrm{CH}$ conserves energy in the network by merging data.

The dynamic $\mathrm{CH}$ selection method for wireless sensor networks uses an estimation algorithm to overcome the imbalance in energy consumption by electing a new $\mathrm{CH}$ based on the ratio of the remaining and average energy of the node on the network from the dead time of the node [26]. In this study, the monitoring area was distributed through a mesh algorithm. The relocation of the area after the sensor node's death in the monitoring area should be considered.

Improved energy-efficient $\mathrm{CH}$ selection in WSNs (IEECHS) improves the $\mathrm{CH}$ selection technique in LEACH and selects dual $\mathrm{CH}$ [27]. The first $\mathrm{CH}$ is used for data transmission, and the second $\mathrm{CH}$ is used to deduplicate data to reduce the communication cost of the network. The use of two CHs in this study shows better performance than LEACH. However, it may affect data transmission delay.

A related study [28] proposed a distributed $\mathrm{CH}$ rotation mechanism to balance $\mathrm{CH}$ energy consumption. To avoid the energy hole problem of associated with a $\mathrm{CH}$ located far away from the BS, the energy efficiency is improved by designing a dynamic multi-hop routing algorithm for $\mathrm{CH}$ nodes using a proposed distance and energy recognition cost function.

A hybrid hierarchical clustering approach (HHCA) is a proposed clustering method based on a three-layer structure [29]. It uses a centralized grid for head selection at the top level and distributed clustering for head selection at the lowest level. It uses a clustering approach similar to LEACH and re-selects grids and clusters in each round.

Partitioned-based energy efficient-LEACH (PE-LEACH) is a proposed method to reduce energy consumption by avoiding multi-hop communication between the $\mathrm{CH}$ and $\mathrm{BS}$ of sensor nodes with mobile BS [30]. The area of the WSNs is divided into a number of quadrants, and the BS will move according to the quadrant. The $\mathrm{CH}$ is selected based on the residual energy of the node.

IoT-LEACH (I-LEACH) is a proposed method to extend the energy efficiency of WSNs by extending the term of the $\mathrm{CH}$ if the residual energy of the elected $\mathrm{CH}$ is above a threshold [31]. The proposed method shows better performance than $\mathrm{LEACH}$, but it may cause problems associated with energy imbalance in the $\mathrm{CH}$.

Learning automata-based multilevel heterogeneous routing (LA-MHR) is a proposed S-model-based LA for the election of $\mathrm{CHs}$ that uses single-hop and multi-hop transmission to increase energy efficiency [32].

The energy-aware routing protocol (EAP) compares the average residual energy of all neighboring nodes in the $\mathrm{CH}$ selection and uses a new clustering parameter in which the sensor node with the highest residual energy becomes the $\mathrm{CH}$ [33]. In addition, building a routing tree between $\mathrm{CHs}$ to maintain the energy load balance between sensor nodes was proposed. The EAP proposed in this study has better performance than LEACH according to the simulation results.

A previous report proposed a multi-hop energy-neutral clustering (MENC) algorithm [34]. All the $\mathrm{CHs}$ in the network transmit data to the BS by a multi-hop communication method and analyze the data rate and transmission energy consumption to impose energy neutrality constraints. Data throughput increases with MENC compared with LEACH. 
A related study [35] proposed fuzzy logic-based energy efficiency clustering using a fuzzy logic model with residual energy, location suitability, density, compression, and distance from the BS as descriptors. This related study [35] demonstrated improved network lifetime and energy efficiency compared LEACH.

However, EAP, MENC, and related algorithms [35] can affect the limited resources of sensor nodes because each sensor node must maintain information about neighboring nodes and residual energy.

\subsection{Energy Consumption Model}

WSNs are most important for efficient energy use. It is not possible to charge or replace batteries when sensor nodes are sprayed in a given area. That is, the life of the sensor node depends on the life of the battery, which affects the life of the WSNs. The sensor nodes consume energy for sensor detection, computation of data, and transmission and/or reception between sensor nodes. In WSNs, a large mount of energy is used for data transmission, and much research has focused on the routing method for efficient use of transmission energy. The transmission energy model of LEACH is shown in Figure 3 [36].

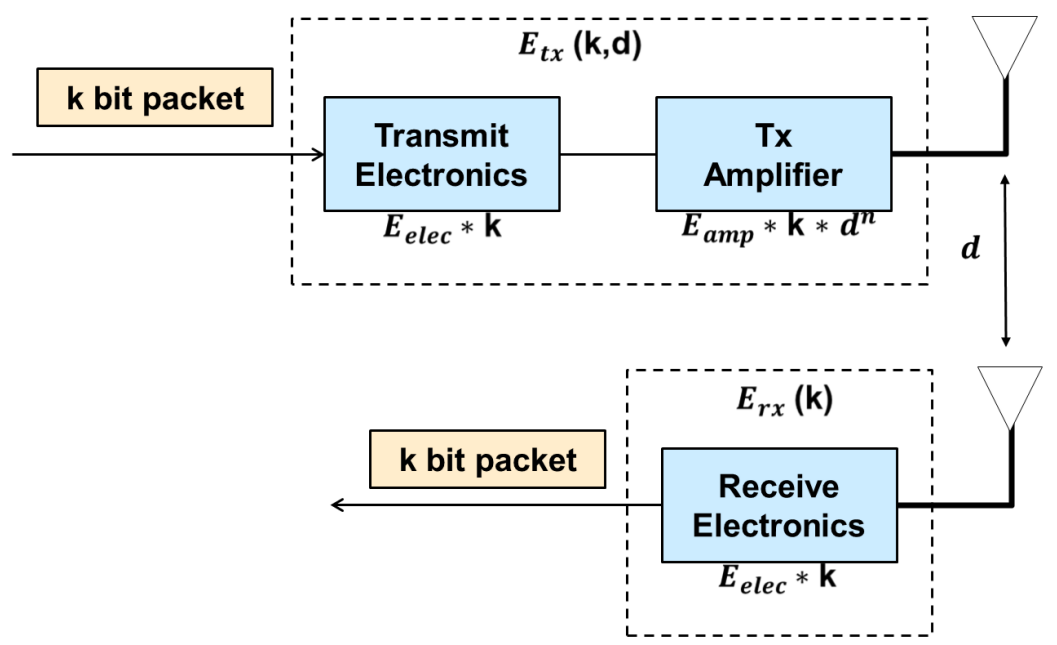

Figure 3. Energy propagation model of low-energy adaptive clustering hierarchy (LEACH).

In Equation (1), the energy consumption $E_{t x}$ required to transmit $k$ bits over the distance $d$ is represented. $E_{\text {elec }}$ is the radio electron energy, and $E_{a m p}$ is the transmitter amplification energy. The amplification energy is divided into a $E_{f s}$ free space propagation model and $E_{m p}$ multipath propagation model according to the critical distance $d_{0}$.

$$
\begin{gathered}
E_{t x}=E_{\text {elec }}(k)+E_{\text {amp }}(k, d), \\
= \begin{cases}k E_{\text {elec }}+k E_{f_{s}} d^{2}, & d<d_{0} \\
k E_{\text {elec }}+k E_{m p} d^{4}, & d \geq d_{0}\end{cases}
\end{gathered}
$$

LEACH distinguishes between the free space propagation model and the multipath propagation model using the $d_{0}$ critical distance defined in Equation (2).

$$
d_{0}=\sqrt{\frac{E_{f s}}{E_{m p}}}
$$

If the transmission distance is smaller than $d_{0}$, a free space propagation model is employed, and if it is large, a multipath propagation model is utilized. In the free space propagation model, power is consumed in proportion to the square of the transmission distance $d_{0}$, and equation $\mathrm{d}$ in the multipath propagation model, power is consumed in proportion to the four square of the transmission distance $d_{0}$. Equation (3) represents the energy consumption when receiving $k$ bits of data. 


$$
E_{r x}=E_{\text {elec }} \times k
$$

\section{LEACH}

LEACH is a hierarchical routing protocol based on clusters in WSNs and selects a $\mathrm{CH}$ among sensor nodes. Then, the $\mathrm{CH}$ collects the data from the neighboring sensor node and transmits it to the BS. LEACH has a set-up phase and a steady-state phase. Figure 4 shows the process of the LEACH protocol.

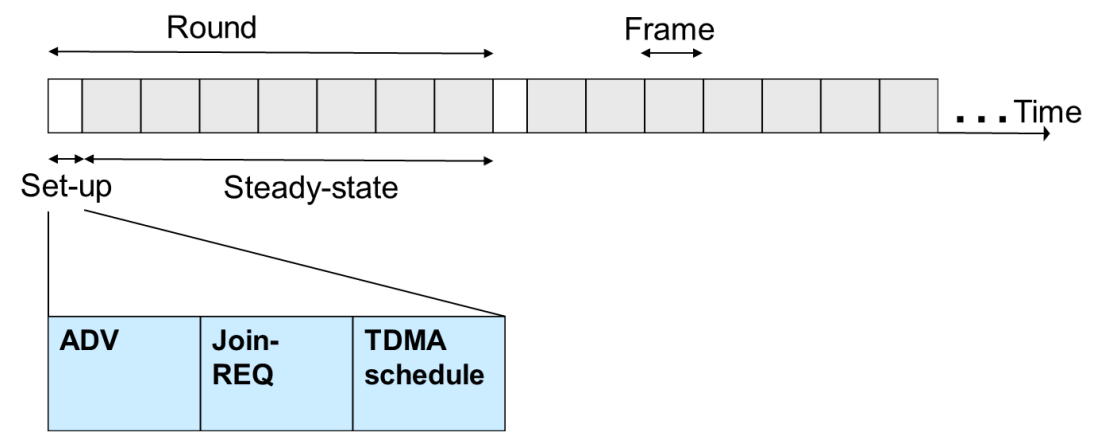

Figure 4. LEACH protocol process.

The two phases constitute one round. The rounds have a constant time interval. After a set time, a new round begins. The BS sends the start message for a new round, and the sensor node executes the set-up phase and the steady-state phase [37,38].

Step 1. Set-up phase

In the set-up phase, $\mathrm{CHs}$ are selected among the sensor nodes, and the $\mathrm{CH}$ s configure the cluster. The $\mathrm{CH}$ is selected according to the probability of the sensor nodes. Each sensor node generates an arbitrary number. Then, an arbitrary number is compared with $T(n)$ in Equation (4).

$$
T(n)= \begin{cases}\left.\frac{P}{1-(r} \bmod \frac{1}{P}\right) & \text { if } n \in G \\ 0 & \text { otherwise }\end{cases}
$$

Here, $G$ is a set of sensor nodes that are not selected as the $\mathrm{CH} . P$ is the probability that the sensor node will be a $\mathrm{CH}$, and $r$ is the current round. If any number of sensor nodes $\mathrm{n}$ is smaller than the value of $T(n)$ and included in $G$, the sensor node $\mathrm{n}$ becomes the $\mathrm{CH}$. The set $G$ initializes at regular intervals. The $\mathrm{CH}$ transmits an advertisement message (ADV) to the surrounding sensor nodes indicating that it is a $\mathrm{CH}$. The surrounding sensor node receiving the ADV compares the signal strength of the ADV. The sensor node sends a join request message (Join-REQ) to the $\mathrm{CH}$ with the highest ADV signal strength. The $\mathrm{CH}$ receives the Join-REQ from surrounding sensor nodes and assigns the time slot to the sensor nodes that have sent Join-REQ. The $\mathrm{CH}$ next sends TDMA schedules to the surrounding nodes. The sensor nodes become member nodes of the $\mathrm{CH}$, and the $\mathrm{CH}$ then completes the cluster configuration.

Step 2. Steady-state phase

The steady-state phase is the data transmission stage. Figure 5 shows the steady-state phase. All member nodes in the cluster transmit data to the $\mathrm{CH}$ according to the TDMA schedule. The $\mathrm{CH}$ merges and compresses the sensor node data and transmits them to the BS. 


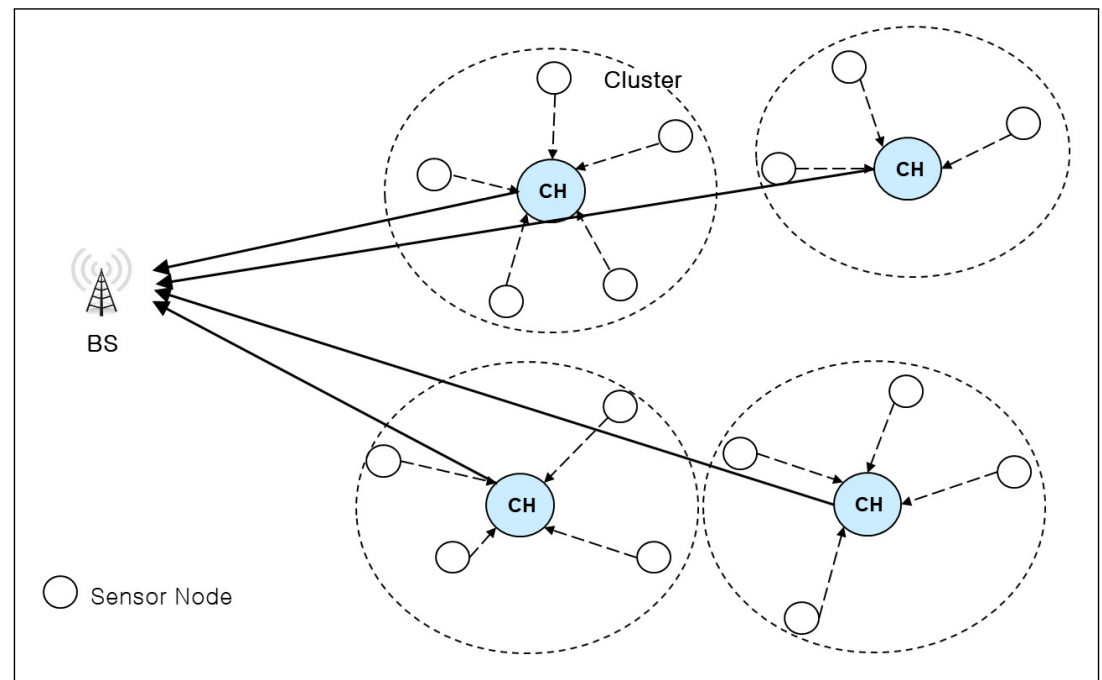

Figure 5. Steady-state phase of LEACH.

Multi-hop LEACH (MH-LEACH) is a routing protocol that uses multi-hop transmission [18,37]. Figure 6 shows the steady-state phase of MH-LEACH.

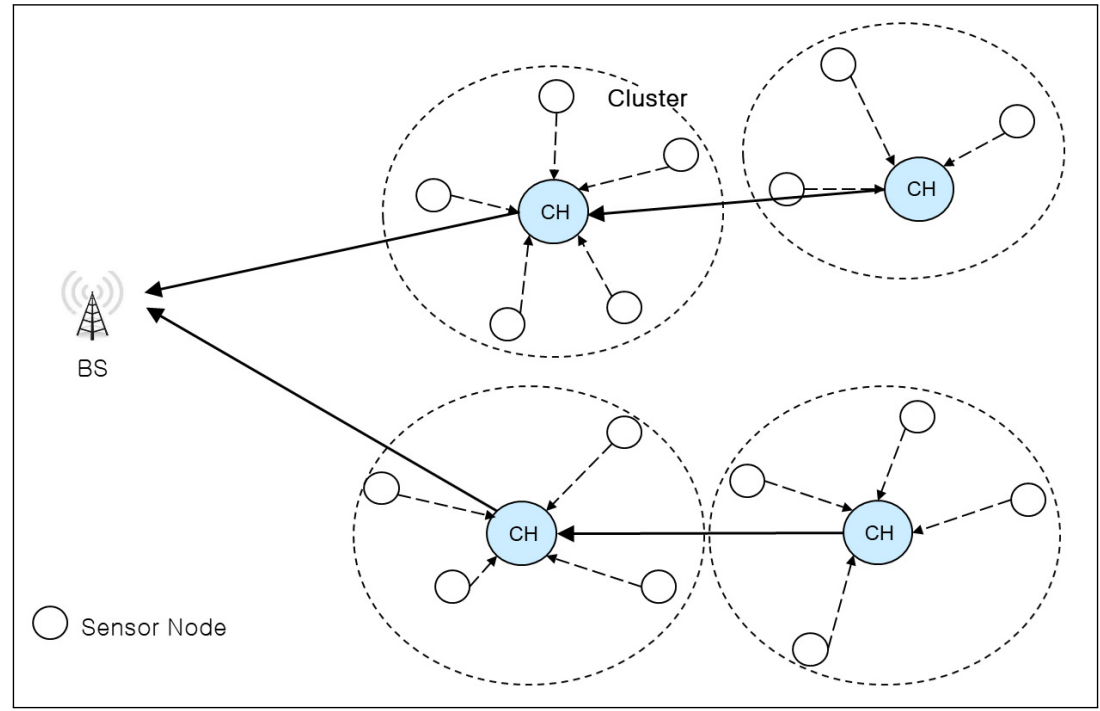

Figure 6. Steady-State phase of a multi-hop LEACH (MH-LEACH).

MH-LEACH increases the transmission energy efficiency of the $\mathrm{CH}$. In LEACH, if the $\mathrm{CH}$ is located far from the BS, the $\mathrm{CH}$ uses a large mount of transmission energy. MH-LEACH constructs an optimal path by selecting multi-hop between $\mathrm{CH}$ and BS. The MH-LEACH designates an upper and lower $\mathrm{CH}$ according to the distance from the $\mathrm{BS}$ position. The lower $\mathrm{CH}$ transmits the data to the upper $\mathrm{CH}$, and the uppermost $\mathrm{CH}$ transmits the data to the BS. MH-LEACH can configure wide-area networks by enabling transmission of data between $\mathrm{CHs}$. In this study, we found a method that can reduce unnecessary energy consumption caused by reverse transmission by improving the LEACH protocol.

\section{D-LEACH}

In this section, we discuss in detail the proposed D-LEACH to improve the energy efficiency of WSNs. D-LEACH is an improved LEACH protocol that reduces unnecessary energy consumption caused by data transmission in the reverse direction relative to the BS. The sensor node of D-LEACH calculates the relative distance using the strength of the signal and determines the transmission 
direction. The BS sends signals to each sensor node. Each sensor node calculates the relative distance to the BS from the strength of the signal. The sensor nodes then determine the direction of transmission by comparing the relative distances of adjacent nodes. D-LEACH uses direct transmission, single-hop transmission, and multi-hop transmission.

The D-LEACH algorithm has four phases.

Step 1. Layer configuration phase.

The BS collects the relative distance information for each sensor node in the WSNs region. The WSN consist of layers according to the relative distance of each sensor node.

Step 2. $\mathrm{CH}$ probability calculation for the layer.

The selection probability of each sensor node for the $\mathrm{CH}$ depends on its layer.

Step 3. Set-up phase.

In this step, the cluster is configured. The sensor node is selected as the $\mathrm{CH}$ using the probability. Sensor nodes not selected as the $\mathrm{CH}$ become member nodes of the $\mathrm{CH}$ by comparing the relative distance. A sensor node that fails to select a $\mathrm{CH}$ that meets the conditions becomes a normal node. Clustering consists of a $\mathrm{CH}$ and member nodes.

Step 4. Steady-state phase.

In this step, data are transmitted from the sensor node and from the $\mathrm{CH}$ to the BS. The member nodes send data to the $\mathrm{CH}$. The $\mathrm{CH}$ merges and compresses the data and sends them to the upper $\mathrm{CH}$ near the BS. Data are sent to the BS via the upper $\mathrm{CH}$. The normal node transmits directly to the BS.

Steps 1 and 2 are performed once in the early stages of WSNs, and Steps 3 and 4 are repeated at regular intervals until all sensor nodes die. Figure 7 shows the steps of the D-LEACH. We explain each step in detail.

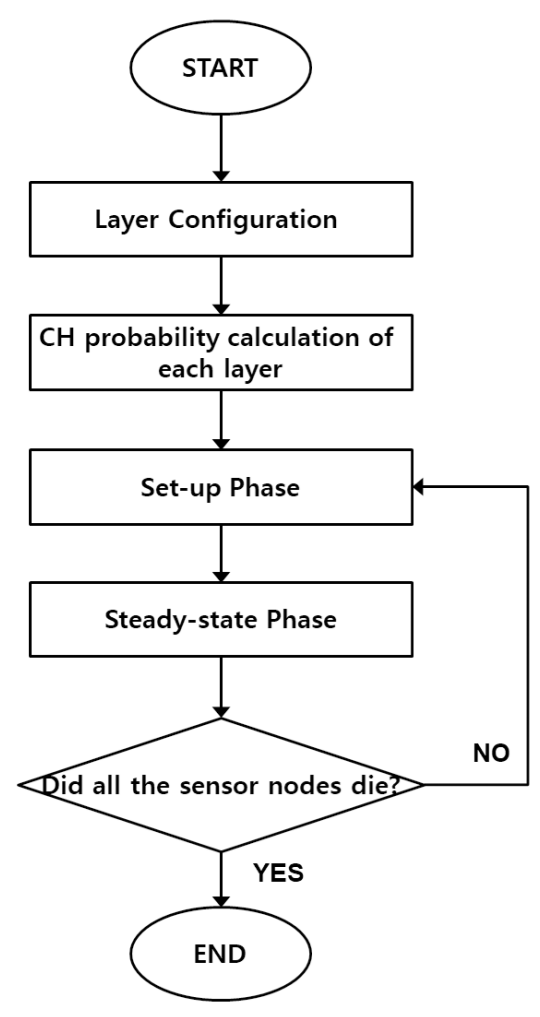

Figure 7. Steps of Direction-based LEACH (D-LEACH). 


\subsection{Layer Configuration Phase}

D-LEACH configures layers of different sizes based on the relative distances of the sensor nodes. Figure 8. shows the layer structure of D-LEACH.

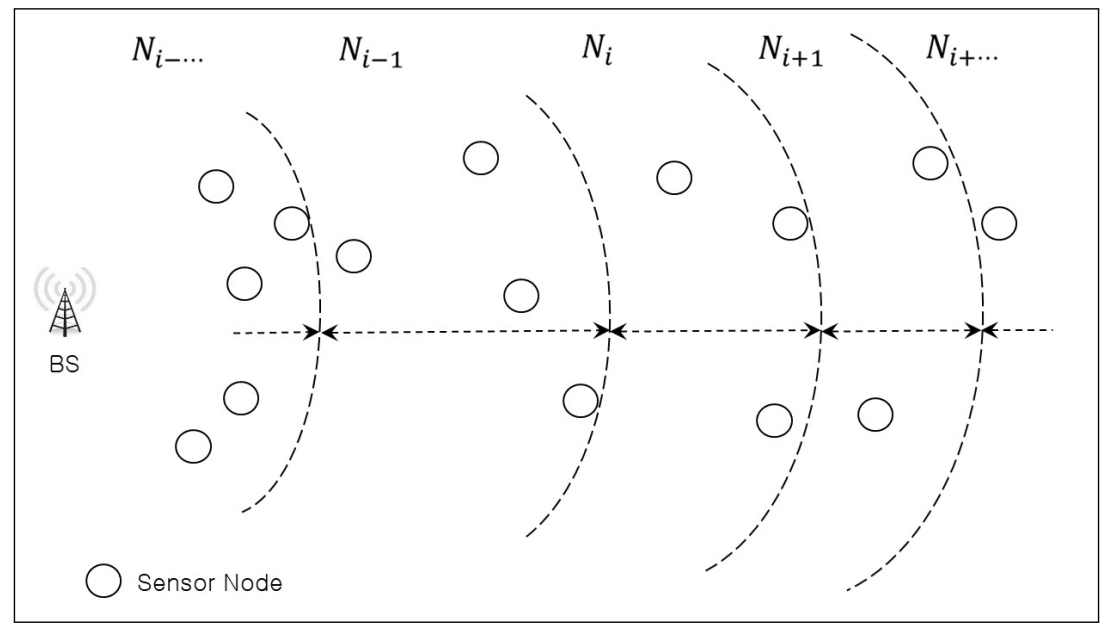

Figure 8. Layer configuration of D-LEACH.

Each sensor node transmits a message including the ID information to the BS. The BS calculates the relative distance of each sensor node based on the strength of the signal and stores the ID of the sensor node. The BS constructs layers of different sizes using the distance of the nearest sensor node and the distance of the farthest sensor node. The sensor nodes located close to the BS are included in the upper layer, and the sensor nodes located far away from the BS are included in the lower layer. The number of sensor nodes included in the layer decreases from the upper layer to the lower layer. The number of sensor nodes in layer $i$ is expressed as $N_{i}$ in Equation (5).

$$
N i=N \times \frac{k-(i-1)}{\sum_{j=1}^{k} j}
$$

The number of sensor nodes in the network is $N$, the total number of layers is $k$, and the layer number is $i$.

\section{2. $\mathrm{CH}$ Probability Calculation for Layers}

Each layer has a different $\mathrm{CH}$ selection probability. For the sensor node to transmit data in the direction in which the BS is located, the $\mathrm{CH}$ selection probability of the upper layer is high, and the $\mathrm{CH}$ selection probability of the lower layer is low. The BS calculates $W_{i}$ as the $\mathrm{CH}$ selection probability value of each layer, as shown in Equation (6).

$$
W i= \begin{cases}P\left(1+\frac{k-(i-1)}{k}\right) & \text { when } i \neq k \\ P & \text { when } i=k\end{cases}
$$

$P$ is the probability value that the sensor node is selected as CH in the WSN, the total number of layers is $k$, and the layer number is $i$. The BS transmits the layer information of each sensor node and the $\mathrm{CH}$ selection probability values of each layer to each sensor node. Each sensor node stores its layer information and the $\mathrm{CH}$ selection probability value. 


\subsection{Set-Up Phase}

The BS relays the start of the round to all sensor nodes. Each sensor node that receives the start message selects a $\mathrm{CH}$ using the $\mathrm{CH}$ probability in Equation (7).

$$
T(n)= \begin{cases}\frac{W i}{1-W i \times\left(r \bmod \frac{1}{W i}\right)} & \text { if } n \in G i \\ & \text { En }>\text { Initial Energy } \times 0.1 \\ 0 & \text { otherwise }\end{cases}
$$

The sensor node's layer number is $i$, and $G_{i}$ is the set of sensor nodes in the $i$ layer not yet selected as $\mathrm{CH}$. Further, $W_{i}$ is the $\mathrm{CH}$ selection probability value of the layer $i$, as in Equation (6), and $r$ is the current round. The sensor node $\mathrm{n}$ is included in $G_{i}$ for the calculation $T(n)$ using $W_{i}$ of its layer and $r . E_{n}$ is the remaining energy of sensor node $\mathrm{n}$. If $E_{n}$ of sensor node $\mathrm{n}$ included in $G_{i}$ is less than $10 \%$, it is excluded from the $\mathrm{CH}$ election candidate. Each sensor node generates an arbitrary number. Then, this number is compared with $T(n)$. If $T(n)$ is larger than the arbitrary number generated by the sensor node $\mathrm{n}$, the sensor node $\mathrm{n}$ becomes the $\mathrm{CH}$.

The $\mathrm{CH}$ transmits an ADV including relative distance information between itself and the BS to the surrounding sensor nodes. Figure 9 shows the ADV of the $\mathrm{CH}$. The ADV message contains the ADV header, ID of the sensor node, ID of the $\mathrm{CH}$, and information regarding the distance to the $\mathrm{BS}$.

\begin{tabular}{|l|l|l|l|}
\hline ADV HEADER & SENSOR ID & CH ID & Distance to the BS \\
\hline
\end{tabular}

Figure 9. Advertisement message (ADV) of cluster header $(\mathrm{CH})$.

Each sensor node that receives the ADV compares the relative distance and selects a $\mathrm{CH}$ to which Join-REQ is transmitted. Figure 10 shows the $\mathrm{CH}$ selection process for the sensor node.

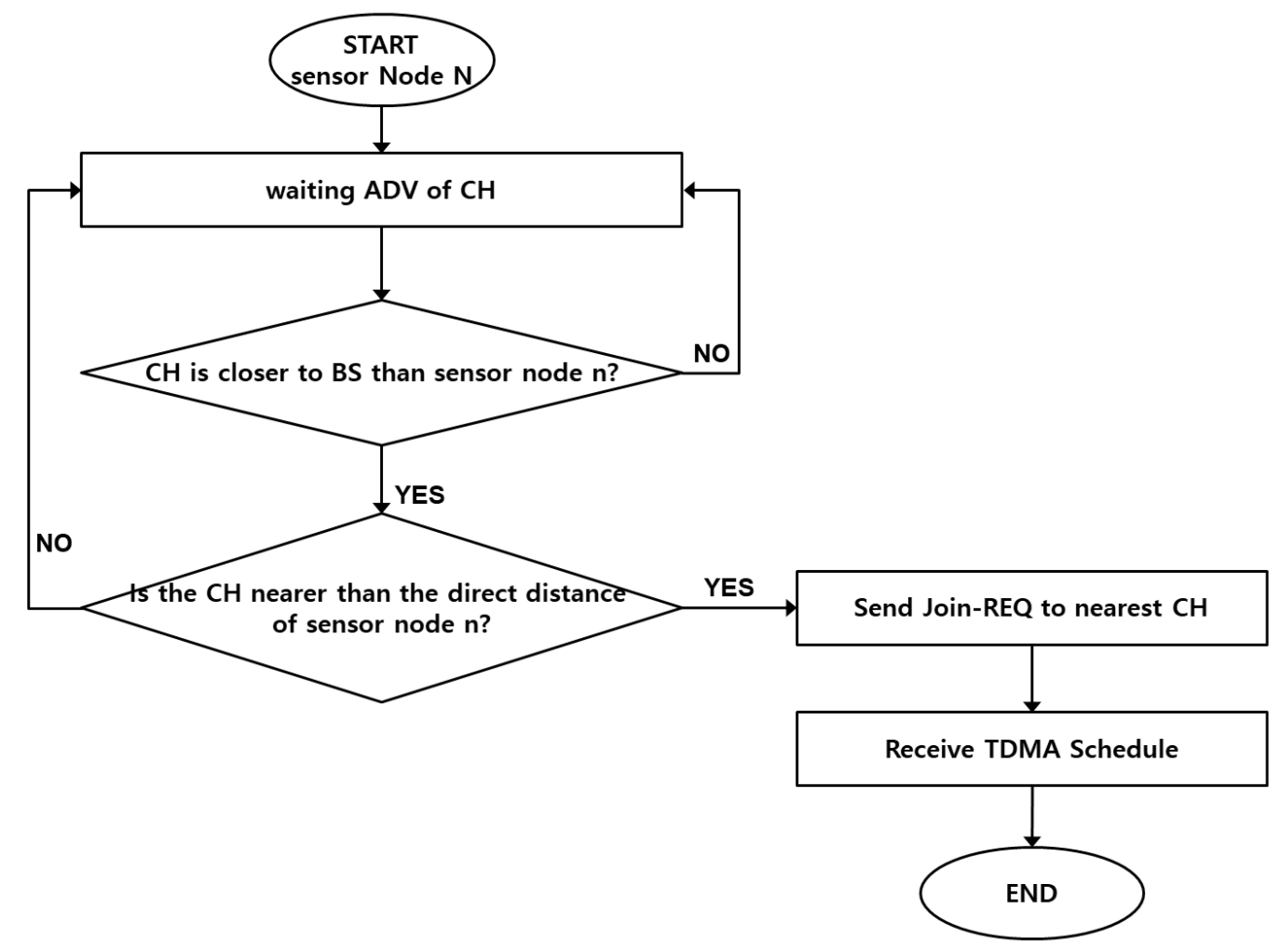

Figure 10. Cluster configuration of sensor nodes. 
(a) The sensor node calculates the distance from the $\mathrm{CH}$ using the reception strength of the ADV.

(b) The sensor node compares the BS distance information of the ADV with its direct transmission distance information and selects $\mathrm{CHs}$ located close to the BS.

(c) The sensor node selects $\mathrm{CHs}$ that have a shorter distance than the direct transmission distance among the $\mathrm{CHs}$ located close to the BS.

(d) The sensor node transmits Join-REQ to the $\mathrm{CH}$ with the shortest transmission distance among the $\mathrm{CHs}$ of (c).

If there is no $\mathrm{CH}$ satisfying this condition, the sensor node does not transmit Join-REQ. The $\mathrm{CH}$ assigns a time slot to the sensor node that transmits Join-REQ and transmits the TDMA schedule. The sensor node receiving the TDMA schedule becomes a member node of the $\mathrm{CH}$, and the cluster configuration is completed.

\subsection{Steady-State Phase}

When the setup phase is completed, data transmission begin. Figure 11 shows the data transfer process of the sensor node.

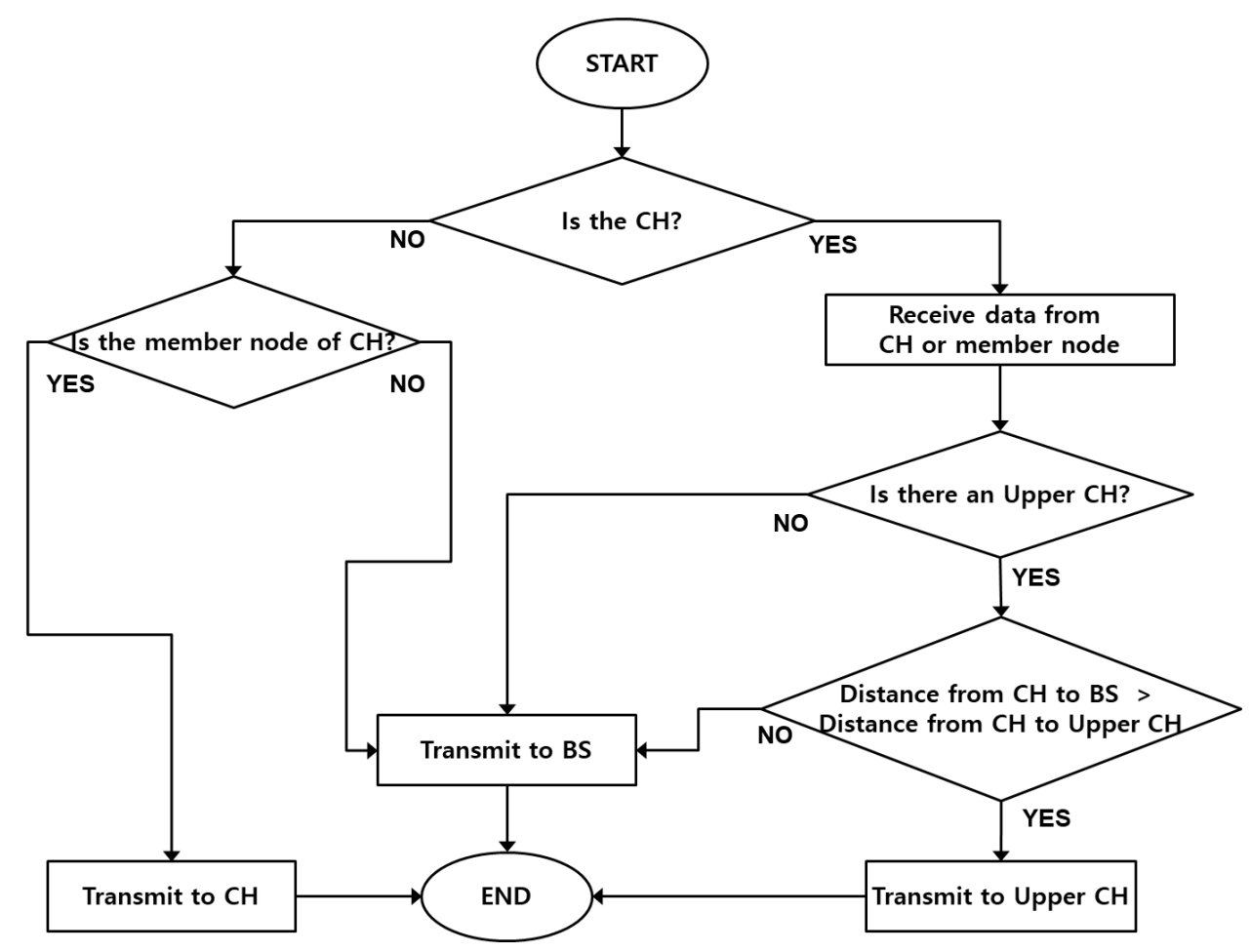

Figure 11. Data transmission process of the sensor node.

D-LEACH distinguishes the $\mathrm{CH}$ according to the distance from the BS. The $\mathrm{CH}$ located close to the $\mathrm{BS}$ is the upper $\mathrm{CH}$, whereas the $\mathrm{CH}$ farther from the $\mathrm{BS}$ is the lower $\mathrm{CH}$. The algorithm uses direct transmission, single-hop transmission, and multi-hop transmission utilizing relative distances. The member nodes of the $\mathrm{CH}$ transmit data to the $\mathrm{CH}$. The sensor nodes, not the member nodes of the $\mathrm{CH}$, transmit directly to the $\mathrm{BS}$. The $\mathrm{CH}$ transmits data to the nearest upper $\mathrm{CH}$ that is closer to the BS than itself and a shorter distance than the direct transmission distance to the BS. If there is no upper $\mathrm{CH}$ corresponding to this condition, the $\mathrm{CH}$ transmits directly. Figure 12 shows the steady-state of D-LEACH. 


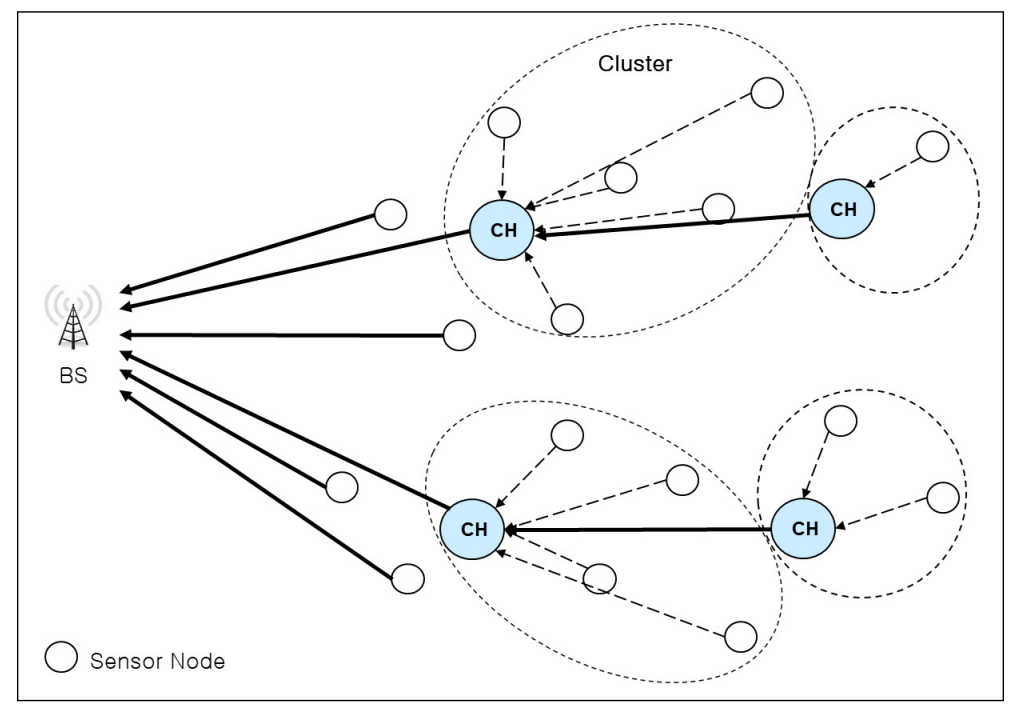

Figure 12. Steady-state of D-LEACH.

\section{Performance Analysis}

WSNs are widely used in IoT systems, and the energy efficiency of sensor nodes affects the network performance of IoT systems. D-LEACH aims to improve the energy efficiency by transferring data in consideration of the direction of data transmission.

In this section, the performance of D-LEACH is compared with that of LEACH and MH-LEACH. We considered a fixed number of sensors and a square field. To verify D-LEACH performance using MATLAB 2014a, 100 sensor nodes was randomly distributed on networks of various sizes $\left(100 \times 100 \mathrm{~m}^{2}, 200 \times 200 \mathrm{~m}^{2}\right.$, and $\left.300 \times 300 \mathrm{~m}^{2}\right)$. The initial energy of the sensor node is $0.5 \mathrm{~J}$, and the sensor node is the same under various conditions. The BS is placed at the bottom left $(0,0)$. The simulation parameters are listed in Table 1. Each network was simulated 20 times in 3500 rounds. The energy consumption model in Section 2.2 was used to evaluate the energy consumption of the sensor nodes.

Table 1. Parameters of the simulation.

\begin{tabular}{cc}
\hline Parameter & Value \\
\hline Number of Sensor nodes & 100 nodes \\
Initial Energy & $0.5 \mathrm{~J}$ \\
Data Size & $2000 \mathrm{bit}$ \\
Transfer Energy $\left(E_{\text {elec }}\right)$ & $50 \mathrm{~nJ} / \mathrm{bit}$ \\
Transmitter Amplifier Energy $\left(E_{f s}\right)$ & $10 \mathrm{pj} / \mathrm{bit} / \mathrm{m}^{2}$ \\
Transmitter Amplifier Energy $\left(E_{m p}\right)$ & $0.0013 \mathrm{pJ} / \mathrm{bit} / \mathrm{m}^{4}$ \\
Merge Energy $\left(E_{d a}\right)$ & $5 \mathrm{~nJ} / \mathrm{bit}$ \\
Number of Layer & $4 \mathrm{Layer}$ \\
Time of Simulation & $3500 \mathrm{rounds}$ \\
Network Size & $100 \times 100 \mathrm{~m}^{2}, 200 \times 200 \mathrm{~m}^{2}$ and $300 \times 300 \mathrm{~m}^{2}$ \\
\hline
\end{tabular}

There are five assumptions about the routing algorithm being evaluated.

(1) Sensor nodes are randomly placed in the network. The position of the deployed sensor node does not change.

(2) All sensor nodes in the network have the same sensing technology, computing technology, and communication technology.

(3) All sensor nodes have limited energy and are not charged.

(4) In the event of a failed packet transmission resulting from a link error, retransmissions of packets are not considered. 
(5) The BS periodically transmits the starting message of the round for data collection, and the sensor node transmits the data according to the routing algorithm.

In this study, the lifetime of the network, the energy efficiency and the packet reception rate of the BS were used as indicators of algorithm performance for evaluation.

(1) The lifetime of the network: the duration of the operation of WSNs from the beginning to the death of the first node (FND). This Indicates the stable state of the network.

(2) Energy efficiency: amount of energy used in a stable network, including the total amount of energy used in a stable network and the amount of energy used in one round.

(3) Packet-received ratio: The number of packets received by the BS while the network is stable.

\subsection{Lifetime of WSNs}

Figures 13-15 show that D-LEACH was superior to the other protocols in terms of network lifetime. When the network size was $100 \times 100 \mathrm{~m}^{2}$, the network lifetime of D-LEACH was $1.5 \%$ and $0.6 \%$ greater, respectively, relative to LEACH and MH-LEACH, as shown in Figure 13. When the network size was $200 \times 200 \mathrm{~m}^{2}$, the network lifetime of the proposed algorithm increased by $452.2 \%$ and $15.6 \%$, respectively, relative to LEACH and MH-LEACH, as shown in Figure 14 . When the network size was $300 \times 300 \mathrm{~m}^{2}$, an increase of $1168.3 \%$ and $111.9 \%$, respectively, for D-LEACH compared with the other two algorithms, as shown in Figure 15. The main reason for these results is that by determining the direction of data transmission for the sensor nodes, the energy retention rate of the sensor nodes increases and prolongs the lifetime of the network because it reduces unnecessary energy consumption caused by reverse transmission.

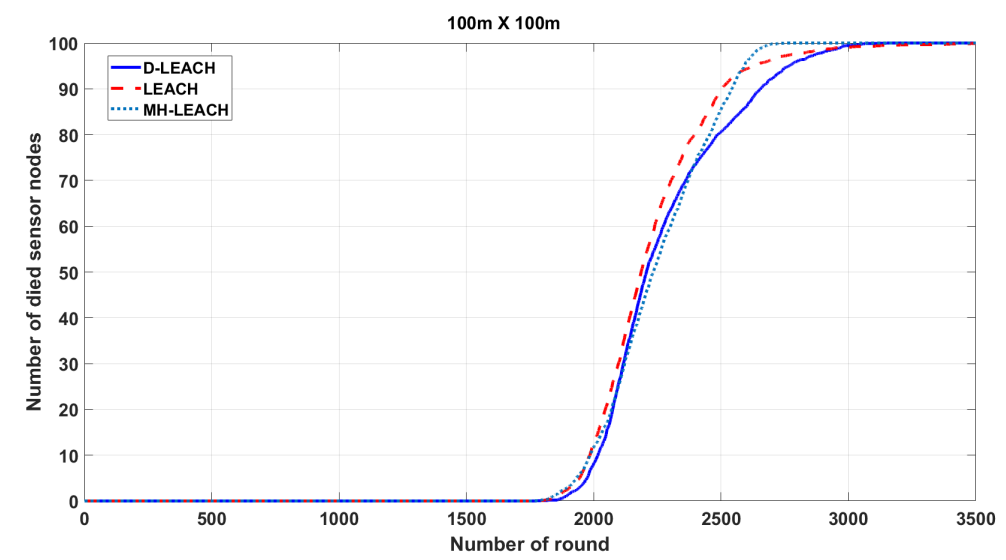

Figure 13. The number of dead sensor nodes in wireless sensor networks (WSNs) $\left(100 \times 100 \mathrm{~m}^{2}\right)$.

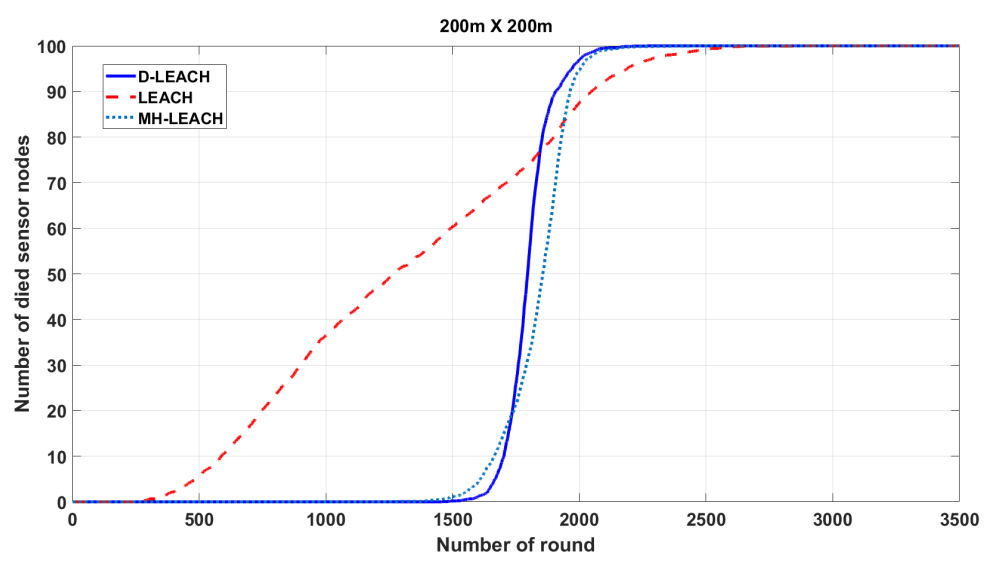

Figure 14. The number of dead sensor nodes in WSNs $\left(200 \times 200 \mathrm{~m}^{2}\right)$. 


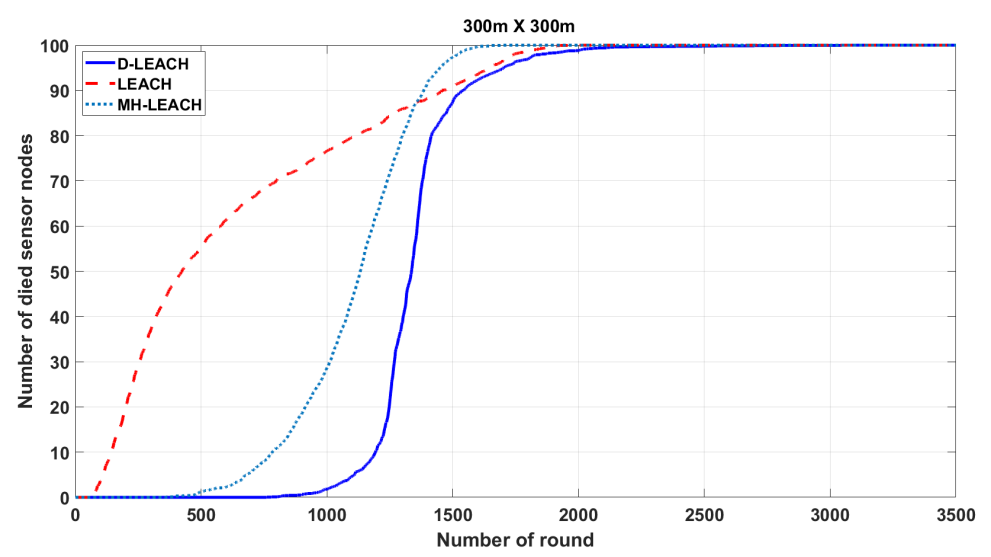

Figure 15. The number of dead sensor nodes in WSNs $\left(300 \times 300 \mathrm{~m}^{2}\right)$.

The network lifetime simulation results regarding the time to FND are shown in Figure 16 for various network sizes. The figure also shows that the FND time for D-LEACH was later than that of LEACH and MH-LEACH. For a network size of $300 \times 300 \mathrm{~m}^{2}$, the network lifetime of D-LEACH was significantly extended compared with that of the LEACH and MH-LEACH protocols. Table 2 shows that the network lifetime of D-LEACH is longer than that of other protocols.

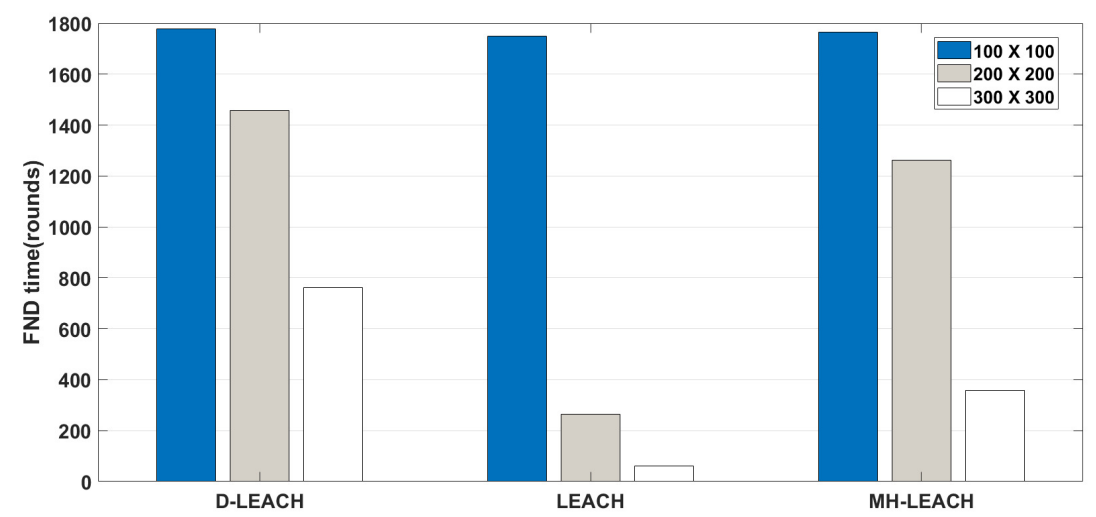

Figure 16. Time of occurrence of death of the first node (FND).

Table 2. FND time ratio according to network size.

\begin{tabular}{cccc}
\hline (FND Time Radio Based on $\left.\mathbf{1 0 0} \times \mathbf{1 0 0} \mathbf{~}^{\mathbf{2}}\right)$ & $\mathbf{1 0 0} \times \mathbf{1 0 0} \mathbf{~}^{\mathbf{2}}$ & $\mathbf{2 0 0} \times \mathbf{2 0 0} \mathbf{~ m}^{\mathbf{2}}$ & $\mathbf{3 0 0} \times \mathbf{3 0 0} \mathbf{~}^{\mathbf{2}}$ \\
\hline D-LEACH & 1777 & 1458 & 761 \\
& $(100 \%)$ & $(82 \%)$ & $(43 \%)$ \\
LEACH & 1750 & 264 & 60 \\
& $(100 \%)$ & $(15 \%)$ & $(3 \%)$ \\
MH-LEACH & 1766 & 1261 & 359 \\
& $(100 \%)$ & $(71 \%)$ & $(20 \%)$ \\
\hline
\end{tabular}

\subsection{Energy Efficiency}

Figure 17 shows the average energy consumption per round in the network stability period. When the network size was $100 \times 100 \mathrm{~m}^{2}$, the energy efficiency of D-LEACH increased by $13.5 \%$ and $2.7 \%$, respectively, relative to LEACH and MH-LEACH. When the network size was $200 \times 200 \mathrm{~m}^{2}$, the energy efficiency of the proposed algorithm increased by $64.5 \%$ and $13.3 \%$, respectively, relative to LEACH and MH-LEACH. When the network size was $300 \times 300 \mathrm{~m}^{2}$, an increase of $86 \%$ and $33.8 \%$, respectively, can be observed compared with the other two algorithms. Therefore, the D-LEACH has higher energy efficiency than the other protocols. 


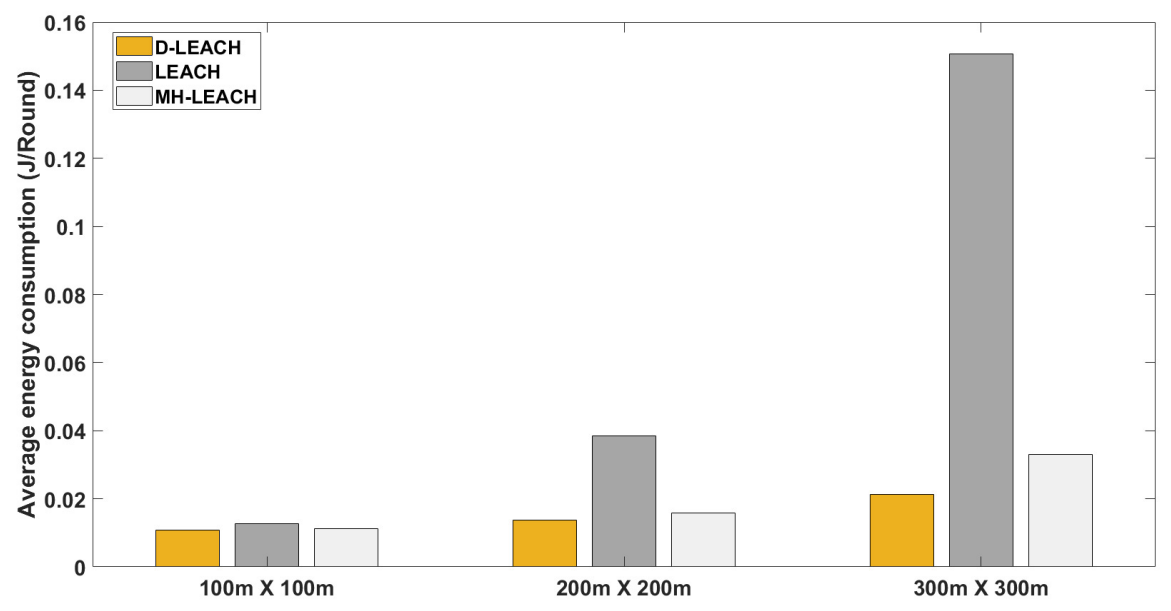

Figure 17. The average amount of energy used per round.

Figures 18-20 show the energy consumption of the network, from which it is evident that the D-LEACH had lower consumption energy, indicating that the network could survive for a longer period of time.

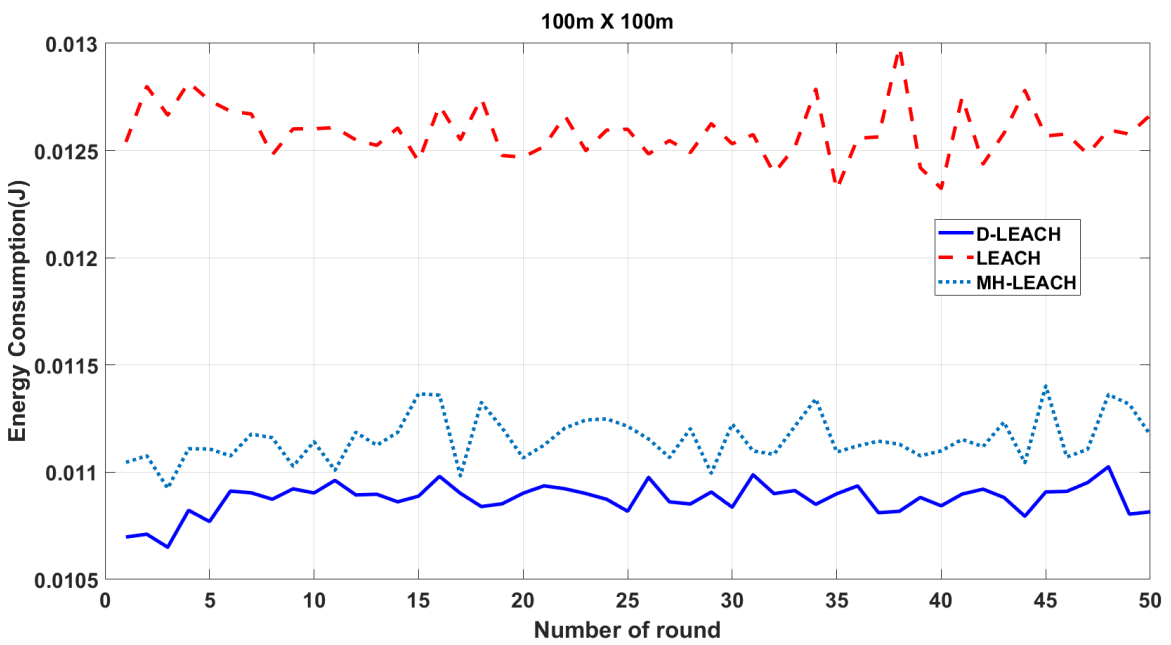

Figure 18. Energy consumption $\left(100 \times 100 \mathrm{~m}^{2}\right)$.

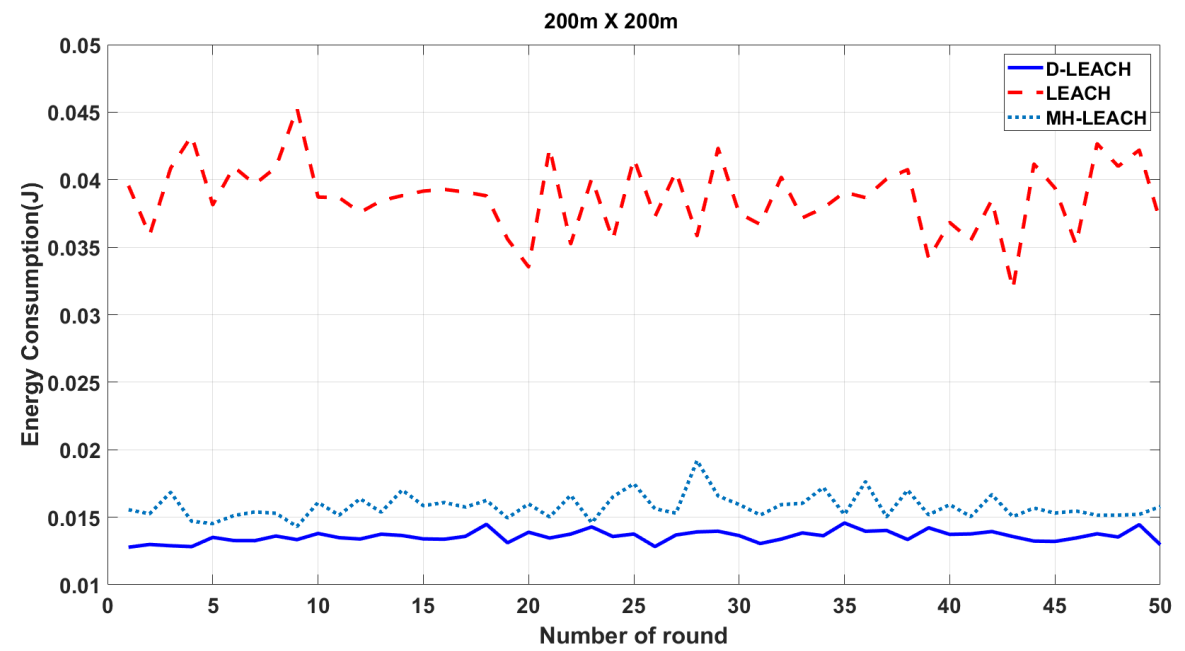

Figure 19. Energy consumption $\left(200 \times 200 \mathrm{~m}^{2}\right)$. 


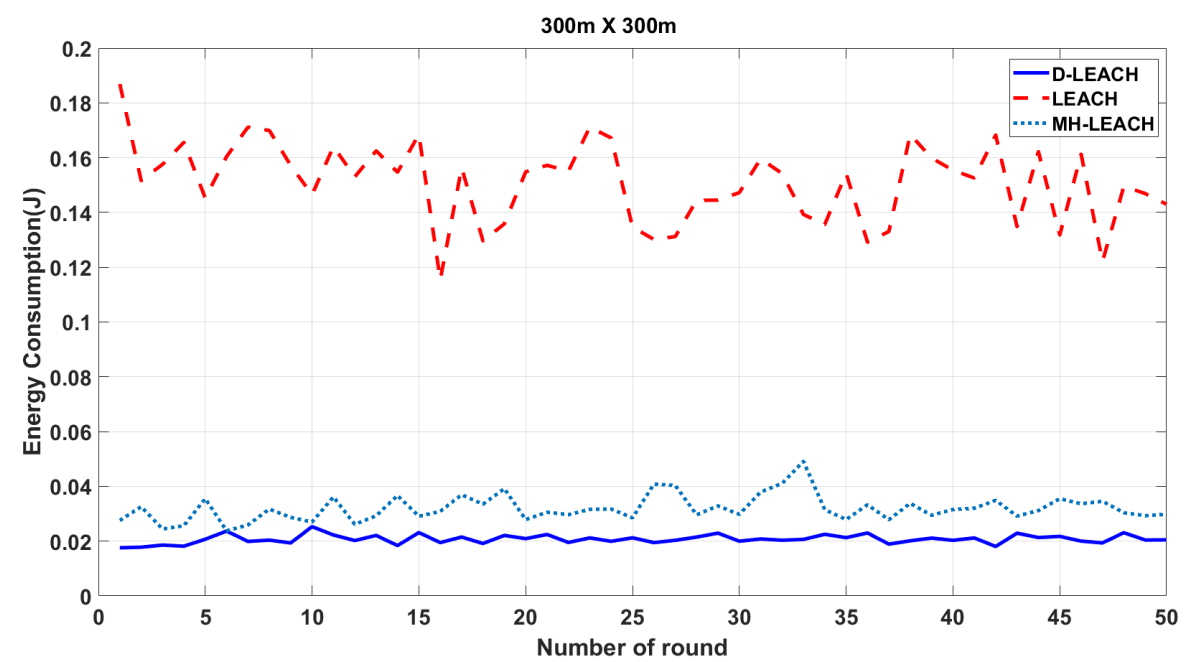

Figure 20. Energy consumption $\left(300 \times 300 \mathrm{~m}^{2}\right)$.

\subsection{Packet-Received Ratio}

Figure 21 shows the number of packets received by the BS while the network status is stable. The network throughput of D-LEACH increased relative to those of LEACH and MH-LEACH. This is because the D-LEACH algorithm adopts the direction of data transmission to reduce unnecessary energy consumption. Thus, the nodes have a longer survival time. The time that the nodes survive in the network is longer than that of other algorithms, and the number of packets received by the BS also increases.

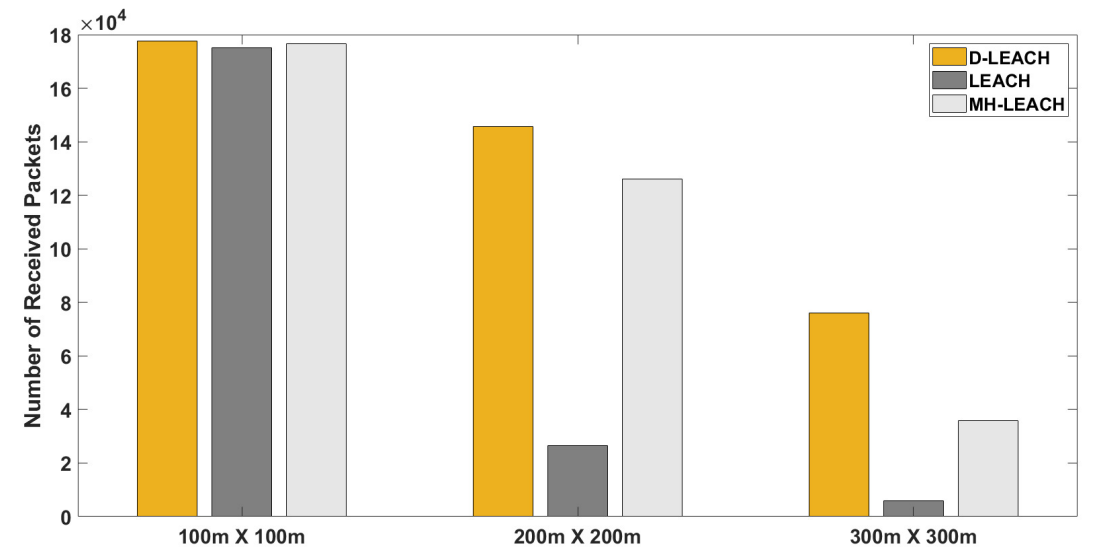

Figure 21. The number of packets received by base station (BS) while the network is stable.

\subsection{Discussion}

The D-LEACH protocol was found to be superior to LEACH and MH-LEACH in terms of network life, power consumption, and BS data reception. The reasons for this are as follows.

(1) LEACH and MH-LEACH transmit data to the $\mathrm{CH}$, closest to the sensor node, and do not consider the transmission distance or direction of transmission. Even if the BS is located close to the sensor node, the data are sent to the distant $\mathrm{CH}$. D-LEACH determines the transmission direction by comparing the relative distances of the sensor node (to the BS, and the sensor node) to the $\mathrm{CH}$. It transmits data to the $\mathrm{CH}$ near the $\mathrm{BS}$ and saves energy consumed by direct transmission if there is no $\mathrm{CH}$ that meets these conditions. This shows excellence even when the network size increases. Sensor nodes consume a large amount of energy in data transmission, which is related to the lifespan of sensor nodes. Saving transmission energy extends the life of the sensor node, which extends the lifetime of the network. 
(2) During $\mathrm{CH}$ selection, LEACH and MH-LEACH do not check the residual energy of the sensor node. If a sensor node with a small residual energy is selected as the $\mathrm{CH}$, it consumes a large amount of energy in data merging, compression, and transmission, and causing the sensor node to die quickly. D-LEACH ensures that sensor nodes with residual energy below the critical residual energy are not selected as the $\mathrm{CH}$, thus maintaining the energy balance of the sensor nodes.

The lifetime of WSNs affects the network performance of the IoT system, and the lifetime of WSNs is determined by the lifetime of the sensor nodes. D-LEACH is thus superior to LEACH and MH-LEACH in terms of network lifetime, power consumption, and data throughput.

\section{Conclusions}

IoT systems consist of wired and wireless communication, various heterogeneous devices, and various software. WSNs are widely used in the design of IoT systems. WSNs consist of sensor nodes with limited energy and the performance of sensor nodes affects IoT systems. Extending the lifetime of WSNs by increasing the transmission energy efficiency of sensor nodes is an important task. LEACH is a well-known protocol for WSNs. Many studies have been conducted that have revised the limits of LEACH to enhance the performance of WSNs. In this study, we proposed the D-LEACH protocol to reduce unnecessary transmission energy consumption caused by the reverse transmission when the sensor node is located between the BS and the $\mathrm{CH}$. D-LEACH is a routing protocol that transmits data using the relative distance based on the RSSI between the sensor node and the BS. D-LEACH divides the network area into several layers using the relative distance between the sensor node and the BS. Each layer has a $\mathrm{CH}$ selection probability. The sensor nodes become the $\mathrm{CH}$ using the $\mathrm{CH}$ selection probability in the layer. The sensor nodes compare the relative distances of $\mathrm{CH}$ and BS it becomes a member of the $\mathrm{CH}$ or becomes a normal node. When the cluster configuration of WSNs is completed, the sensor node data are transmitted to the BS using direct transmission, single-hop transmission, or multi-hop transmission.

To evaluate the performance of D-LEACH, we confirmed the lifetime, average energy consumption, and network throughput of WSNs according to the network size. Performance comparison showed that D-LEACH extends the lifetime of WSNs relative to LEACH and MH-LEACH. In addition, the average energy consumption of D-LEACH is less than that of LEACH and MH-LEACH. In particular, when the size of the WSNs increases, D-LEACH shows better performance. This shows that the energy balance of sensor nodes in D-LEACH is superior to that of LEACH and MH-LEACH. D-LEACH reduces the consumption associated with reverse transmission energy and increases the efficiency of data transmission energy, improving the network performance of IoT systems.

In this study, we focused on reducing the consumption of transmission energy in WSNs. The security and reliability of the data were also considered. Future research tasks will determine how to secure enhance security and reliability by proposing cryptographic transmission of data in D-LEACH and how to reduce packet size to increase energy efficiency.

Author Contributions: Formal analysis, K.M.N. and J.S.P.; funding acquisition, J.S.P.; methodology, K.M.N. and J.S.P.; project administration, J.S.P.; resources, K.M.N. and J.H.P.; writing-original draft, K.M.N.; writing-review and editing, K.M.N, J.H.P. and J.S.P. All authors have read and agreed to the published version of the manuscript.

Funding: This research was supported by the Basic Science Research Program through the National Research Foundation of Korea (NRF) funded by the Ministry of Education (NRF2017R1D1A1B03035833).

Conflicts of Interest: The authors declare no conflict of interest.

\section{References}

1. Musaddiq, A.; Zikria, Y.B.; Hahm, O.; Yu, H.; Bashir, A.K.; Kim, S.W. A survey on resource management in IoT operating systems. IEEE Access 2018, 6, 8459-8482. [CrossRef]

2. Pan, M.-S.; Chen, C.-J. Intuitive control on electric devices by smartphones for smart home environments. IEEE Sens. J. 2016, 16, 4281-4294. [CrossRef] 
3. Chunli, L. Intelligent transportation based on the Internet of Things. In Proceedings of the 2012 2nd International Conference on Consumer Electronics, Communications and Networks (CECNet), Yichang, China, 21-23 April 2012; pp. 360-362.

4. Jones, C.; Warburton, B.; Carver, J.; Carver, D. Potential applications of wireless sensor networks for wildlife trapping and monitoring programs. Wildl. Soc. Bull. 2015, 39, 341-348. [CrossRef]

5. Xu, L.; Collier, R.; O'Hare, G.M. A survey of clustering techniques in WSNs and consideration of the challenges of applying such to 5G IoT scenarios. IEEE Internet Things J. 2017, 4, 1229-1249. [CrossRef]

6. Wang, C.; Zheng, W.; Bertino, E. Provenance for wireless sensor networks: A survey. Data Sci. Eng. 2016, 1, 189-200. [CrossRef]

7. Gnanambigai, J.; Rengarajan, D.N.; Anbukkarasi, K. Leach and its descendant protocols: A survey. Int. J. Commun. Comput. Technol. 2012, 1, 15-21.

8. Akyildiz, I.F.; Su, W.; Sankarasubramaniam, Y.; Cayirci, E. Wireless sensor networks: A survey. Comput. Netw. 2002, 38, 393-422. [CrossRef]

9. Rhim, H.; Tamine, K.; Abassi, R.; Sauveron, D.; Guemara, S. A multi-hop graph-based approach for an energy-efficient routing protocol in wireless sensor networks. Hum. Cent. Comput. Inf. Sci. 2018, 8, 1-21. [CrossRef]

10. Wan, R.; Xiong, N. An energy-efficient sleep scheduling mechanism with similarity measure for wireless sensor networks. Hum. Cent. Comput. Inf. Sci. 2018, 8, 18. [CrossRef]

11. Lee, J.-H.; Shin, B.-S. SensDeploy: Efficient sensor deployment strategy for real-time localization. Hum. Cent. Comput. Inf. Sci. 2017, 7, 36. [CrossRef]

12. Yu, B.; Choi, W.; Lee, T.; Kim, H. Clustering Algorithm Considering Sensor Node Distribution in Wireless Sensor Networks. J. Inf. Process. Syst. 2018, 14, 926-940. [CrossRef]

13. Moustafa, K.E.; Hafid, H. Self-Identification of Boundary's Nodes in Wireless Sensor Networks. J. Inf. Process. Syst. 2017, 13, 128-140.

14. Ari, A.A.A.; Yenke, B.O.; Labraoui, N.; Damakoa, I.; Gueroui, A. A power efficient cluster-based routing algorithm for wireless sensor networks: Honeybees swarm intelligence based approach. J. Netw. Comput. Appl. 2016, 69, 77-97. [CrossRef]

15. Gupta, I.; Riordan, D.; Sampalli, S. Cluster-head election using fuzzy logic for wireless sensor networks. In Proceedings of the 3rd Annual Communication Networks and Services Research Conference (CNSR'05), Halifax, NS, Canada, 16-18 May 2005; pp. 255-260.

16. Al-Karaki, J.N.; Kamal, A.E. Routing techniques in wireless sensor networks: A survey. IEEE Wirel. Commun. 2004, 11, 6-28. [CrossRef]

17. Heinzelman, W.R.; Chandrakasan, A.; Balakrishnan, H. Energy-efficient communication protocol for wireless microsensor networks. In Proceedings of the 33rd annual Hawaii International Conference on System Sciences, Maui, HI, USA, 7 January 2000; Volume 12, p. 10.

18. Marhoon, H.A.; Mahmuddin, M.; Nor, S.A. DCBRP: A deterministic chain-based routing protocol for wireless sensor networks. SpringerPlus 2016, 5, 2035. [CrossRef]

19. Zhang, D.-G.; Wang, X.; Song, X.-D.; Zhang, T.; Zhu, Y.-N. A new clustering routing method based on PECE for WSN. EURASIP J. Wirel. Commun. Netw. 2015, 2015, 162. [CrossRef]

20. Diop, A.; Qi, Y.; Wang, Q.; Hussain, S. An advanced survey on secure energy-efficient hierarchical routing protocols in wireless sensor networks. arXiv 2013, arXiv:1306.4595.

21. Younis, O.; Fahmy, S. HEED: A hybrid, energy-efficient, distributed clustering approach for ad hoc sensor networks. IEEE Trans. Mob. Comput. 2004, 3, 366-379. [CrossRef]

22. Kulothungan, K.; Ganapathy, S.; Indra Gandhi, S.; Yogesh, P.; Kannan, A. Intelligent secured fault tolerant routing in wireless sensor networks using clustering approach. Int. J. Soft Comput. 2011, 6, 210-215. [CrossRef]

23. Li, Y.; Yu, N.; Zhang, W.; Zhao, W.; You, X.; Daneshmand, M. Enhancing the performance of LEACH protocol in wireless sensor networks. In Proceedings of the 2011 IEEE Conference on Computer Communications Workshops (INFOCOM WKSHPS), Shanghai, China, 10-15 April 2011; pp. 223-228.

24. Li, J.; Silva, B.N.; Diyan, M.; Cao, Z.; Han, K. A clustering based routing algorithm in IoT aware Wireless Mesh Networks. Sustain. Cities Soc. 2018, 40, 657-666. [CrossRef]

25. Feng, X.; Zhang, J.; Ren, C.; Guan, T. An unequal clustering algorithm concerned with time-delay for Internet of Things. IEEE Access 2018, 6, 33895-33909. [CrossRef] 
26. Jia, D.; Zhu, H.; Zou, S.; Hu, P. Dynamic cluster head selection method for wireless sensor network. IEEE Sens. J. 2015, 16, 2746-2754. [CrossRef]

27. Jesudurai, S.A.; Senthilkumar, A. An improved energy efficient cluster head selection protocol using the double cluster heads and data fusion methods for IoT applications. Cogn. Syst. Res. 2019, 57, 101-106. [CrossRef]

28. Wang, Z.; Qin, X.; Liu, B. An energy-efficient clustering routing algorithm for WSN-assisted IoT. In Proceedings of the 2018 IEEE Wireless Communications and Networking Conference (WCNC), Barcelona, Spain, 15-18 April 2018; pp. 1-6.

29. Lee, J.-S.; Kao, T.-Y. An improved three-layer low-energy adaptive clustering hierarchy for wireless sensor networks. IEEE Internet Things J. 2016, 3, 951-958. [CrossRef]

30. Mohapatra, H.; Rath, A.K. Fault tolerance in WSN through PE-LEACH protocol. IET Wirel. Sens. Syst. 2019, 9, 358-365. [CrossRef]

31. Behera, T.M.; Samal, U.C.; Mohapatra, S.K. Energy-efficient modified LEACH protocol for IoT application. IET Wirel. Sens. Syst. 2018, 8, 223-228. [CrossRef]

32. Tanwar, S.; Tyagi, S.; Kumar, N.; Obaidat, M.S. LA-MHR: Learning automata based multilevel heterogeneous routing for opportunistic shared spectrum access to enhance lifetime of WSN. IEEE Syst. J. 2018, 13, 313-323. [CrossRef]

33. Liu, M.; Cao, J.; Chen, G.; Wang, X. An energy-aware routing protocol in wireless sensor networks. Sensors 2009, 9, 445-462. [CrossRef]

34. Yang, L.; Lu, Y.; Zhong, Y.; Wu, X.; Yang, S.X. A multi-hop energy neutral clustering algorithm for maximizing network information gathering in energy harvesting wireless sensor networks. Sensors 2016, 16, 26. [CrossRef]

35. Hamzah, A.; Shurman, M.; Al-Jarrah, O.; Taqieddin, E. Energy-efficient fuzzy-logic-based clustering technique for hierarchical routing protocols in wireless sensor networks. Sensors 2019, 19, 561. [CrossRef]

36. Bae, S.-K. Power Consumption Analysis of Prominent Time Synchronization Protocols for Wireless Sensor Networks. JIPS 2014, 10, 300-313. [CrossRef]

37. Xiangning, F.; Yulin, S. Improvement on LEACH protocol of wireless sensor network. In Proceedings of the 2007 International Conference on Sensor Technologies and Applications (SENSORCOMM 2007), Valencia, Spain, 14-20 October 2007; pp. 260-264.

38. Heinzelman, W.B. Application-Specific Protocol Architectures for Wireless Networks; Massachusetts Institute of Technology: Cambridge, MA, USA, 2000. 